ARTICLE

\title{
Millisecond dynamics of an unlabeled amino acid transporter
}

Tina R. Matin 1,2,4, George R. Heath (10 1,2,4, Gerard H. M. Huysmans (1) 2, Olga Boudker ${ }^{2,3}$ \& Simon Scheuring (1,2区 $^{1,2}$

Excitatory amino acid transporters (EAATs) are important in many physiological processes and crucial for the removal of excitatory amino acids from the synaptic cleft. Here, we develop and apply high-speed atomic force microscopy line-scanning (HS-AFM-LS) combined with automated state assignment and transition analysis for the determination of transport dynamics of unlabeled membrane-reconstituted $\mathrm{Glt}_{\mathrm{Ph}}$, a prokaryotic EAAT homologue, with millisecond temporal resolution. We find that $\mathrm{Glt}_{\mathrm{Ph}}$ transporters can operate much faster than previously reported, with state dwell-times in the $50 \mathrm{~ms}$ range, and report the kinetics of an intermediate transport state with height between the outward- and inwardfacing states. Transport domains stochastically probe transmembrane motion, and reversible unsuccessful excursions to the intermediate state occur. The presented approach and analysis methodology are generally applicable to study transporter kinetics at system-relevant temporal resolution.

\footnotetext{
${ }^{1}$ Department of Anesthesiology, Weill Cornell Medicine, 1300 York Avenue, New York, NY 10065, USA. ${ }^{2}$ Department of Physiology and Biophysics, Weill Cornell Medicine, 1300 York Avenue, New York, NY 10065, USA. ${ }^{3}$ Howard Hughes Medical Institute, Weill Cornell Medicine, New York, NY 10065 , USA.

${ }^{4}$ These authors contributed equally: Tina R. Matin, George R. Heath. ${ }^{凶}$ email: sis2019@med.cornell.edu
} 
E xcitatory amino acid transporters (EAATs) are a family of integral membrane proteins that are essential in the mammalian central nervous system (CNS). This family of proteins assures efficient neurotransmission and prevents glutamate-mediated neurotoxic effects by regulating extracellular transmitter levels of neurons and glial cells surrounding synapses. Through EAA removal from the synaptic cleft, EAATs reset the synapse for the next round of neurotransmission. Malfunction of EAATs results in various neurological pathologies such as poor recovery after stroke and neurodegenerative diseases ${ }^{1-4}$. Expression of glutamate transporters is not exclusive to the CNS, but also occurs in other organs such as kidney (EAAT3) or the retina where EAAT5 mediates glutamate-dependent chloride influx into cells $s^{5-7}$. This vital family of transporters symports three sodium $\left(\mathrm{Na}^{+}\right)$ions and one proton $\left(\mathrm{H}^{+}\right)$along with the EAA, and antiports one potassium $\left(\mathrm{K}^{+}\right)$ion $^{8,9}$.

Glt $_{\mathrm{Ph}}$ is an archaeal homolog of EAATs from Pyrococcus horikoshii. Glt $\mathrm{Ph}_{\mathrm{Ph}}$ shares $\sim 37 \%$ amino acid sequence identity with human EAAT2, and has a high structural similarity with human EAAT1 ${ }^{10}$ (RMSD: $1.5 \AA$ ) and with a neutral amino acid transporter ASCT2 ${ }^{11}$. Glt $t_{\mathrm{Ph}}$ is an excellent model system to study EAATs: the first structure (outward-facing) was solved in $2004^{12}$, and since then, about a dozen structures describe various conformational states (outward- and inward-facing, and intermediate) and substrate binding occupancies ${ }^{13}$. Glt $\mathrm{Ph}_{\mathrm{Ph}}$ is a bowlshaped homo-trimer comprising a central trimerization domain and three peripheral transport domains (Fig. S1). Each transport domain consists of four transmembrane (TM) helices and two helical hairpins (HP1, HP2) that are essential in substrate binding. HP2 has been shown to play the key role in the gating process on both, the extracellular ${ }^{14-20}$ and the intracellular sides of the membrane ${ }^{20-23}$.

Glt $_{\mathrm{Ph}}$, like EAATs, symports three $\mathrm{Na}^{+}$-ions (down their electrochemical concentration gradient from the extracellular to the intracellular space), and L-aspartate (Asp, against the concentration gradient) across the membrane. In contrast to EAATs, Glt $t_{\mathrm{Ph}}$ does not require $\mathrm{H}^{+}$-symport or $\mathrm{K}^{+}$-antiport to complete the transport cycle $14,22,24$. While other transporters function by the rocker-switch (e.g., $\mathrm{LacY}^{25}$ ) or gated-pore (e.g., LeuT ${ }^{26}$ ) mechanisms, Glt $t_{\mathrm{Ph}}$ displays an "elevator" mechanism, whereby the transport domains move $\sim 1.8 \mathrm{~nm}$ across the membrane ${ }^{12,27-32}$. The transport domain movement is a stochastic and thermally driven process, and each protomer is independent of the others within the trimer 19,27,28,30-33. The "elevator" transport mechanism is general, and prokaryotic and eukaryotic proteins from several families work with "elevator" domains (Table S1, e.g. $3,4,6,34$, for a review see ${ }^{35}$ ).

Glt $_{\mathrm{Ph}}$ transport dynamics have been studied using singlemolecule FRET (smFRET) of tethered transporters in detergent ${ }^{28}$ or vesicles 29,30 , and by high-speed atomic force microscopy (HS-AFM) that visualized the dynamics of unlabeled $\mathrm{Glt}_{\mathrm{Ph}}$ in membrane directly ${ }^{33}$. Although these observations represented tremendous progress in our mechanistic understanding of the transporter, the use of low photon intensity to acquire timeextended FRET traces and the challenge to acquire highly contrasted HS-AFM movies set limits to the spatio-temporal resolution. As a consequence, short-lived events and transport substates may have escaped observation.

Here, we develop and apply HS-AFM ${ }^{36}$ line scanning (HS-AFM$\mathrm{LS}^{37}$ ) on unlabeled molecules and report $\mathrm{Glt}_{\mathrm{Ph}}$ transport domain movements with unprecedented spatial and temporal resolution. Our results represent the most detailed recordings of individual transport domains under close-to-physiological (at ambient temperature and pressure and in membrane) conditions providing insights into rapid state transitions and transport sub-states.

\section{Results}

Reconstitution of Glt $_{\mathrm{Ph}}$ and HS-AFM imaging. Purified Glt $\mathrm{Ph}_{\mathrm{Ph}}$ was reconstituted into vesicles of up to $300 \mathrm{~nm}$ in size. The proteo-liposomes were deposited on freshly cleaved mica and imaged in buffer solution. HS-AFM captured proteo-liposomes surface adsorption and spreading (Fig. 1a and Supplementary Movies 1 and 2). Height histogram analysis documented the vesicle adsorption (Fig. 1b, $t=3 \mathrm{~s}$ ), and reported membranes with a thickness of $\sim 4.5 \mathrm{~nm}$ containing Glt $_{\mathrm{Ph}}$-packed regions with $\sim 7.7$ $\mathrm{nm}$ thickness after spreading (Fig. $1 \mathrm{~b}, t=33 \mathrm{~s}$ ). Proteo-liposomes that spread in close proximity fused into larger patches, attesting membrane fluidity (Supplementary Movies 1 and 2). The height difference of $\sim 3.2 \mathrm{~nm}$ between Glt $_{\mathrm{Ph}}$ domains and bare bilayer resulted in a bimodal height distribution that allowed thresholding and area analysis of the $\mathrm{Glt}_{\mathrm{Ph}}$-packed domains (Fig. 1b, right): $\mathrm{Glt}_{\mathrm{Ph}}$ covered only $\sim 30 \%$ of the total membrane area. Thus, Glt $t_{\mathrm{Ph}}$ preferentially arranges in densely packed domains despite the availability of an extended free membrane. Although the dense packing might be a consequence of the chosen lipid composition that is different from the native environment in Pyrococcus horikoshii ${ }^{38}$, we note that mammalian EAATs cluster too with up to $\sim 5000$ transporters $/ \mu \mathrm{m}^{2}$ in glial membranes around synaptic clefts 39,40 .

High-resolution HS-AFM imaging of these domains resolved the Glt $_{\mathrm{Ph}}$ trimers and the individual protomers within them well (Fig. 1c, d and Supplementary Movie 3), surpassing in clarity and resolution our earlier HS-AFM data ${ }^{33}$. Comparison with $\mathrm{Glt}_{\mathrm{Ph}} \mathrm{X}$ ray structures showed that the vast majority of transporters exposed the extracellular face, where the protomers stand out of membrane $\sim 2 \mathrm{~nm}$ with an inter-protomer distance of $5.7 \mathrm{~nm}$, to the HS-AFM tip (Fig. S2). Indeed, in the high-resolution images (Fig. 1c, d), the $\mathrm{Glt}_{\mathrm{Ph}}$-packed domains are covered with molecules protruding $\sim 2 \mathrm{~nm}$ leaving essentially no space for molecules inserted in the other direction. In agreement with previous studies $^{29,33}$, only a minority of the transporters displayed dynamics, while the others appeared silent and exposed the transport domains to the extracellular side (Fig. 1e). Dynamic protomers were observed at locations distributed over the entire membrane (Supplementary Movie 3). The range of the "elevator" movement in this experiment was $1.2 \pm 0.3 \mathrm{~nm}$, in good agreement with earlier X-ray ${ }^{10}, \mathrm{HS}^{-\mathrm{AFM}^{33}}$, and smFRET $^{28,30}$ data.

High-speed AFM line scanning (HS-AFM-LS): millisecond transporter dynamics. Among the bulk and single-molecule techniques, HS-AFM stands out with its ability to provide realtime structural and dynamical information of single molecules. HS-AFM images label-free molecules under close-tophysiological conditions with $\sim 0.1 \mathrm{~nm}$ vertical and $\sim 1 \mathrm{~nm}$ lateral imaging resolution. Furthermore, HS-AFM has typically $\sim 100 \mathrm{~ms}$ temporal resolution, giving access to structure-dynamics relationship of proteins ${ }^{41}$, though the achievable imaging speed depends on sample characteristics like scan size and surface corrugation. Recently in a quest to achieve higher temporal resolutions, we used HS-AFM line scanning (HS-AFM-LS ${ }^{37}$ ) for the analysis of single-protein dynamics. Line scanning, using a conventional AFM, has been used to study protein-protein interactions earlier ${ }^{42}$. In HS-AFM-LS, the slow-scan axis $(y$ direction) is disabled. Therefore, instead of imaging an $x / y$-area, we scan over one horizontal $x$-line several hundreds to thousands of times per second, thus reaching millisecond temporal resolution $^{37}$. The topographical readouts of this line are stacked one after another, resulting in kymographs of the dynamical behavior of the molecules. Therefore, HS-AFM-LS has between 2 and 3 orders of magnitude higher temporal resolution than HS-AFM 

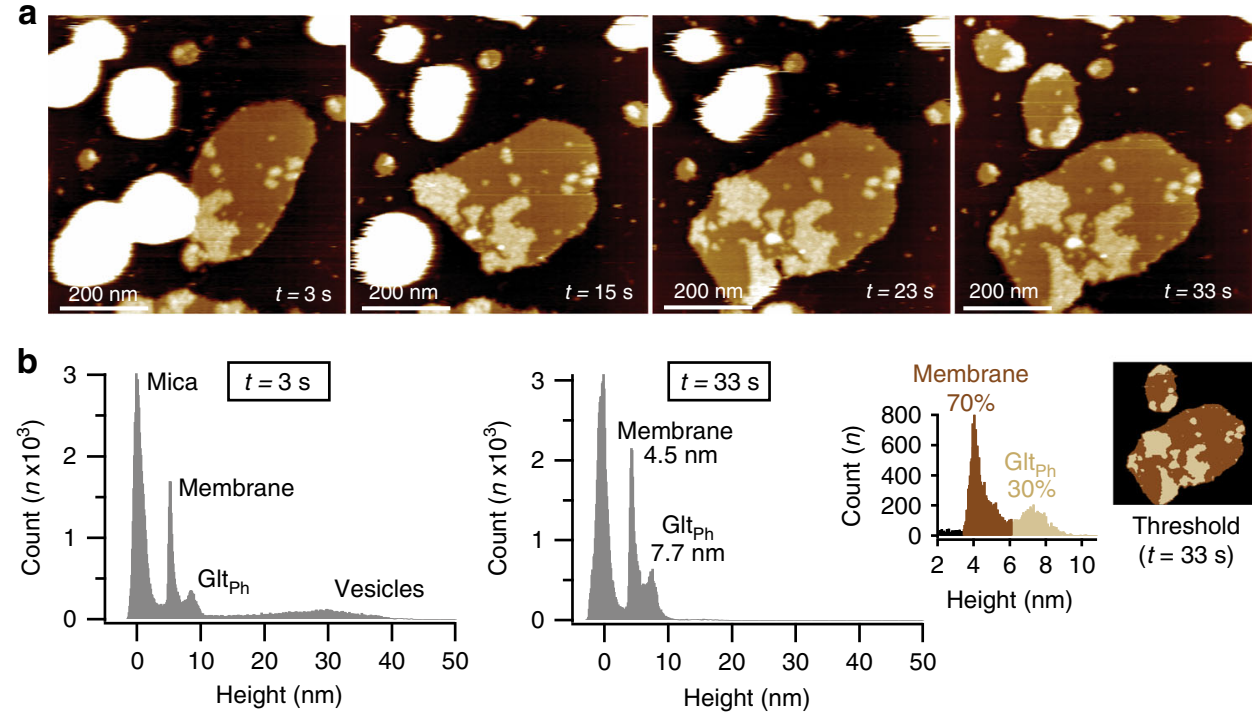

Threshold

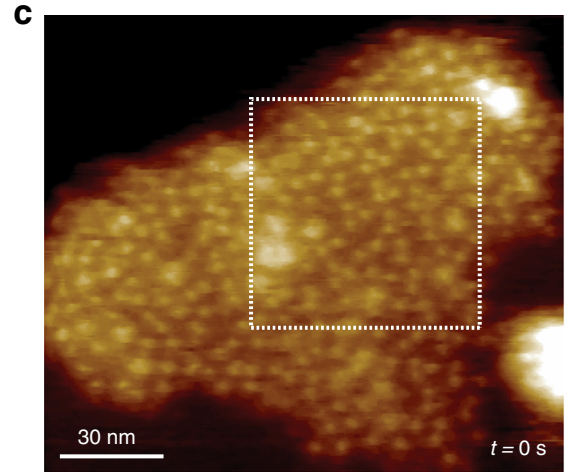

d

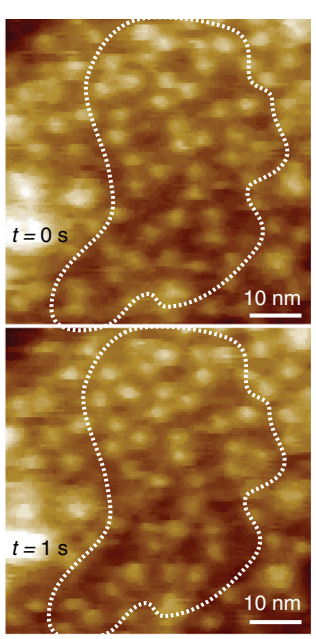

e
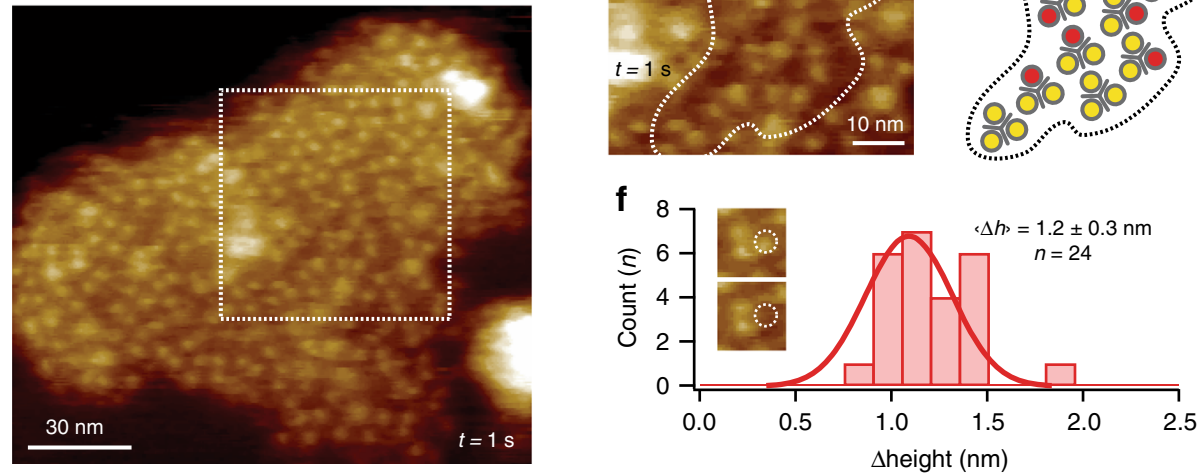

Fig. 1 HS-AFM imaging of $\mathbf{~ G l}_{\mathbf{p h}}$ morphology and activity in membranes. a HS-AFM movie frames of Glt $t_{\text {ph }}$ proteo-liposomes spreading on mica (Supplementary Movies 1 and 2; representative for $>50$ experimental replicates). b Height distribution analysis: initially $(t=3 \mathrm{~s})$ intact vesicles (height: $\sim 30 \mathrm{~nm}$ ) adsorb to the surface (defined height: $\sim 0 \mathrm{~nm}$ ) that eventually $\left(t=33 \mathrm{~s}\right.$ ) spread into membrane sheets (height: $\sim 4.5 \mathrm{~nm}$ ) with Glt $\mathrm{t}_{\mathrm{Ph}}$ domains (height: $\sim 7.7 \mathrm{~nm}$ ). Right: membrane packing analysis with thresholds at $3.5 \mathrm{~nm}<$ membrane $<6.1 \mathrm{~nm}$ and $6.1 \mathrm{~nm}<\mathrm{Glt} \mathrm{thh}_{\mathrm{Ph}}<10 \mathrm{~nm}$. Membranes are $\sim 30 \%$ Glt $t_{\mathrm{Ph}}$-packed. c Consecutive high-resolution images. $\mathbf{d}$ Zoomed areas of the dashed outlines in c showing Glt $\mathrm{t}_{\mathrm{ph}}$ trimers and protomer activity (representative for $>50$ experimental replicates). e Schematic representation of the dashed outlines in $\mathbf{d}$, where yellow and red circles represent outwardand inward-facing protomers, respectively. $\mathbf{f}$ Height change ( $\Delta$ height) distribution of protomer "elevator" motions in $\mathbf{c}$. Mean \pm s.d. are indicated ( $n=24)$. Inset: example protomer (dashed circle).

imaging and should allow the detection of fast transporter dynamics and possible intermediate states that have so far escaped kinetic characterization.

HS-AFM-LS experiments begin as imaging experiments to localize a Glt $t_{\mathrm{Ph}}$ patch and center it in the scan area (Fig. 2a). Disabling the $y$-scan motion engages HS-AFM-LS mode, placing the tip to the central line of the prior imaging frame, and starting repetitive scanning over the protomers located underneath that scan line, thus producing a topography kymograph (Fig. 2b).
Projecting the kymograph in the time axis (Fig. 2c, top) allows plotting an average section profile (Fig. $2 \mathrm{c}$, bottom). After recording the kymograph, another area scan demonstrates the absence of lateral stage drift and preservation of the membrane integrity throughout the experiment (Fig. 2d). Close inspection of the kymograph revealed transport domain movements to the inward-facing state as periods of decreased height along the time axis (Fig. 2e). These excursions were in the $250 \mathrm{~ms}$ range and shorter and, therefore, would have been missed in area imaging. 

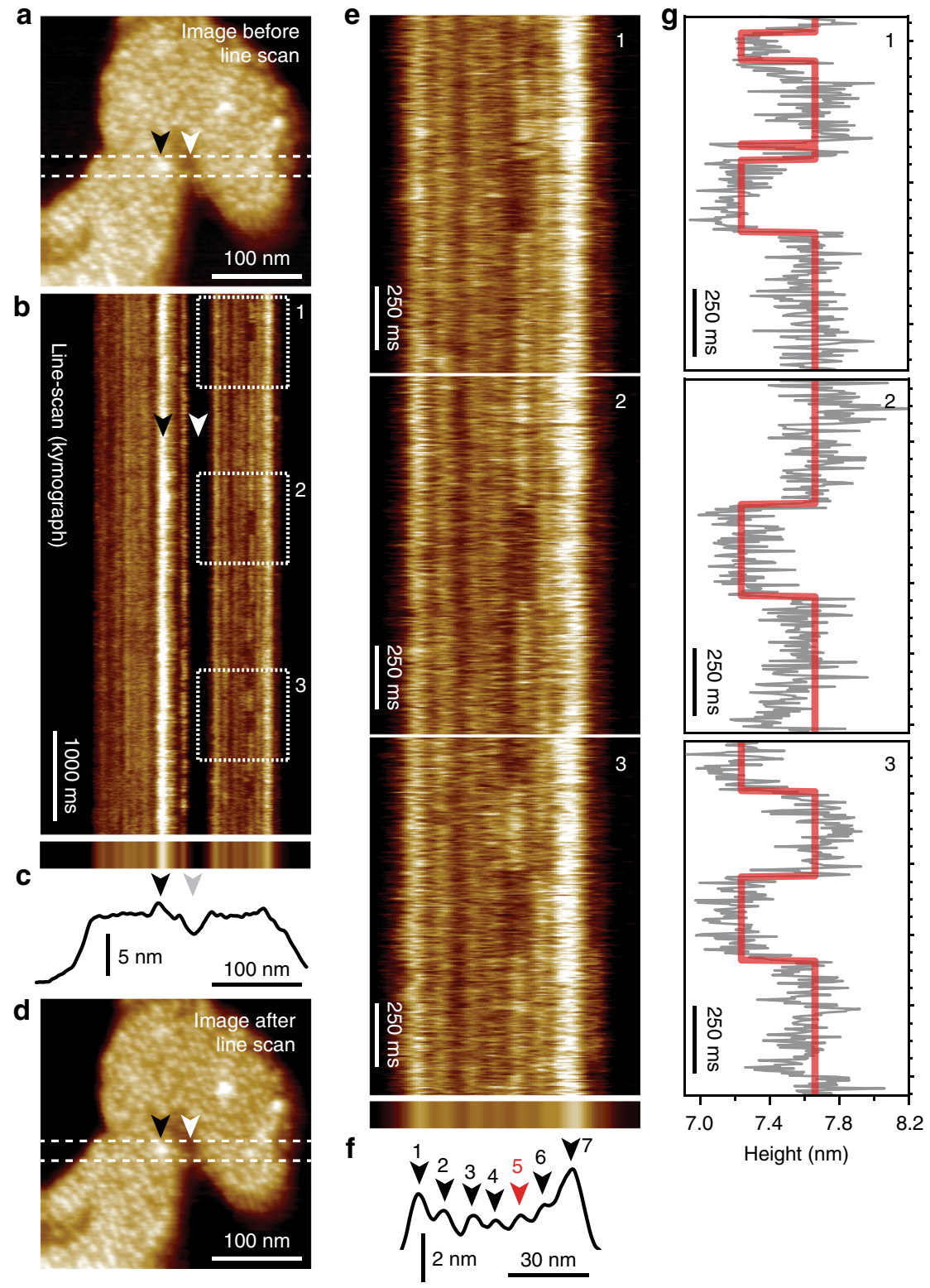

Fig. 2 HS-AFM line scanning (HS-AFM-LS): millisecond temporal resolution of unlabeled transporter dynamics. a HS-AFM image of a membrane packed with Glt $\mathrm{Ph}_{\mathrm{Ph}}$ exposing the extracellular face before HS-AFM-LS (apo condition: $20 \mathrm{mM}$ Tris- $\mathrm{HCl}, \mathrm{pH} 7.5,150 \mathrm{mM} \mathrm{KCl}$ ). Dashed lines indicate the position of the central scan line where subsequent HS-AFM-LS is performed. $\mathbf{b}$ Six seconds of a HS-AFM-LS kymograph with 3.3 ms line acquisition speed. Each transporter domain appears as a vertical line. c Projection (top) and height profile (bottom) of $\mathbf{b}$. d HS-AFM image after HS-AFM-LS. The lateral position of recognizable features in $\mathbf{a}-\mathbf{d}$ are indicated by arrowheads. e One second high-magnification views of dashed regions 1, 2, and 3 in $\mathbf{b}$. Transport domain excursions to the inward-facing state appear as dark dwells along the vertical time axis. $\mathbf{f}$ Projection (top) and height profile (bottom) of e. Arrowheads indicate the position of the seven protomers in the kymograph (red: active protomer \#5). $\mathbf{g}$ Height/time traces (gray) and state fits (red) of the active domain (protomer \#5) in e. This figure is representative of the experimental sequence for the $>50$ replicates analyzed in this work.

Projection in the time axis (Fig. 2f, top) and section analysis showed that in this example, seven protomers were tracked but only protomer \#5 (Fig. 2f, bottom, red arrowhead) displayed activity during observation (Fig. 2e). To analyze the dynamics of the transport domains, a software to track the $x$-positions (Fig. S3), and a state-transitions and -assignment algorithm to fit the vertical movements (Fig. S4) of the protomers over time were developed and applied, respectively (see "Methods"). In HSAFM-LS, the single scan line contours transporter domains at varying relative locations of their topographical structure, and therefore the measured "elevator motion" amplitude varies between protomers (Figs. S5 and 2e). We analyzed in detail how stage drift and molecular motion would impact line scan kymographs (Supplementary Movie 4): while X drift leads to positional shift of the protomers in the kymographs, $\mathrm{Y}$ drift leads to a change of height, because the scan line crosses the protomer at a different position, i.e. height level. Stage drift varies dramatically, from $\sim 1.0$ to $0.02 \mathrm{~nm} / \mathrm{s}$, depending on experimental duration and thermal equilibration ${ }^{37}$. Rotation and fast diffusion of trimers lead to loss of their signal in the kymograph. Thus, activity can be determined from traces displaying no drift, or X drift over extended periods, while Y drift leads to decrease of the signal power and loss of the trace. The dense packing of Glt $t_{\mathrm{Ph}}$ trimers in the membrane typically prevents rotation and diffusion 
of trimers, and the vast majority of kymographs display height/ time traces of protomers that are positionally stable and remain at constant height over tens of seconds. Through the tracking and state assignment approaches, HS-AFM-LS kymographs were transformed into idealized height/time traces that allowed the extraction of transport domain states dwell-times (Fig. 2g).

HS-AFM-LS detects fast transitions and an intermediate state. To characterize Glt $_{\mathrm{Ph}}$ transport-specific dynamics, HS-AFM-LS experiments were performed under relevant environmental conditions, i.e., apo, transport, and inhibitory control conditions. It is, however, important to note that our experimental setup does not allow the establishment of a TM electrochemical gradient. In our experiments, we document the kinetics of the TM passage of either empty (apo) or fully loaded (transport) transporter domains, facing identical conditions on both sides of the membrane.

In the absence of substrate, apo condition, $\mathrm{Glt}_{\mathrm{Ph}}$ was visibly more dynamic and underwent shorter-lived excursions (Fig. 3a), compared to the protein in transport conditions, i.e. in the presence of saturating $\mathrm{Na}^{+}$and Asp concentrations (Fig. 3b). In all conditions, many protomers appeared inactive with their transport domains exposed to the extracellular face (Fig. 3a, b, bottom; see also Fig. 1c). Indeed, only $55 \%$ and $23 \%$ of protomers of the total of 340 and 422 protomers tracked in the kymographs in apo and transport conditions, respectively, displayed dynamics during our observation window, in agreement with previous work $^{29,33}$. In some previous smFRET works transporters interchanged between extended rests in the outward-facing state and bursts of activity $28-30,33$. Here, we rather observe either inactive or active protomers. We attribute the fact that we do not see an interchange of activity modes to the experimental differences as compared to the mentioned smFRET studies. We hypothesize that conformational sub-states of HP2, specific lipid interactions and lipid composition ${ }^{38}$, protein packing, or membrane deformation effects ${ }^{43}$ underlie the different behaviors of individual protomers in our experiments.

Dynamic protomers were characterized by broad and multimodal height distributions. In contrast, the height distributions of non-dynamic protomers were more uniform (Fig. 3, right column). The protomers' heights above membrane and motion amplitude were $>1 \mathrm{~nm}$ in agreement with the transporter domain membrane protrusion height on the extracellular side in the X-ray structures. Glt $t_{\mathrm{Ph}}$ HS-AFM-LS height/time traces recorded in saturating $\mathrm{Na}^{+}$conditions or in the presence of the inhibitor TBOA display little molecular activity, as expected and in agreement with earlier smFRET and HS-AFM studies ${ }^{28,33}$ (Fig. S6 and Table S2). The HS-AFM-LS traces reached 2 min recording length at $3.3 \mathrm{~ms}$ line acquisition speed, and the mean protomer tracking duration was $\sim 23 \mathrm{~s}$ (Fig. 3c), which is mainly limited by experiment-dependent stage drift (Fig. S7). At $3.3 \mathrm{~ms}$ per scan line, we miss transporter activities shorter than $6.6 \mathrm{~ms}$. Reducing the length of the line scan in the HS-AFM-LS experiments will allow to reach $\sim 1 \mathrm{~ms}$ temporal resolution in future studies.

Because HS-AFM-LS contours individual protomers at different relative topographical locations (Fig. S5), each protomer had a different protrusion height above the membrane. Therefore, the height differences between elevator motion states were also different, which, together with the experimental noise, allowed assignment of a signal-to-noise ratio (SNR, see "Methods") to each trace (Fig. 3d). Note, the experimental height noise in HSAFM-LS traces on a bare bilayer is Gaussian and has a subAngstrom standard deviation $(\sigma)$ at millisecond temporal sampling (Fig. S8). Among the 283 height/time traces of active protomers (apo and transport conditions pooled), the state- assignment algorithm suggested that 40 protomers visited a third state with transporter domain height between the inward- and outward-facing states (Fig. 3d). We term this state "intermediate state" in the remainder of the manuscript, but cannot determine whether this state corresponds to an intermediate state previously observed structurally or computationally $23,44-47$. As expected, the state-assignment algorithm mainly assigned three states in traces with elevated SNR $>2$. We tested the performance of the stateassignment algorithm on simulated traces overlaid with varying amounts of noise and found a $93 \%$ transition fit accuracy and an $80 \%$ state-assignment accuracy at SNR $=2$ (Fig. S4). Thus, the intermediate state was assigned, and its kinetics evaluated with confidence. In individual traces with $\mathrm{SNR}>2$, the intermediate state was directly visible to the eye (Fig. 3e, see also Fig. 3a, b).

Intermediate states have been structurally observed in many transporters ${ }^{48,49}$, including $\mathrm{Glt}_{\mathrm{Ph}}{ }^{19}$ and the closely related $\mathrm{Glt}_{\mathrm{Tk}}{ }^{23}$. However, the kinetics of these states remained unexplored in previous Glt $_{\mathrm{Ph}}$ smFRET and HS-AFM data that were interpreted with only the outward- and inward-facing states ${ }^{33}$, with the notable exception of a smFRET study that reported an intermediate FRET-state with a $\sim 1.7 \mathrm{~s}$ time constant ${ }^{30}$. HSAFM-LS combines millisecond temporal resolution with Angstrom vertical resolution (Fig. S8). Thus, HS-AFM-LS surpasses previous approaches in temporal and spatial resolution. Another advantage is that the lateral contouring of each transport domain is reported by several pixels (Fig. 3e, top), and thus, the vertical position is described by several thousands of samples per second, resulting in excellent statistics.

State dwell-times and transition pathways. The Glt $t_{\mathrm{Ph}}$ singlemolecule analysis revealed intricate dynamics with a large variety of molecular behaviors and associated state dwell-times. Earlier smFRET and HS-AFM recordings, at the lower temporal resolution, also reported multiple dwell-time characteristics ${ }^{29,33}$. Here, the dwell-time probability density plots and histograms display multiple kinetic components (Figs. 4a and S9 and Table S2, see "Methods" 50 ) in both the absence and presence of substrates. We attribute the differences between our HS-AFM-LS and earlier smFRET dwell-time distributions to the differences in acquisition bandwidth. The millisecond temporal resolution in HS-AFM-LS allows the detection of short excursions, which if missed would lead to longer dwell assignments by concatenating the two neighboring dwells. In apo conditions, protomers spent on average a similar amount of time in the outward- $(347 \mathrm{~ms})$ and inward- $(328 \mathrm{~ms})$ facing states, while the intermediate state was shorter-lived $(175 \mathrm{~ms})$. In the presence of substrates, the protomers were overall less active and spent longer times in the outward- $(510 \mathrm{~ms})$, inward- $(341 \mathrm{~ms})$, and intermediate $(297 \mathrm{~ms})$ states. In both conditions, the outward- and the inward-facing states dwell-time distributions were best fitted with three components, while the intermediate state displayed simpler dwelltime distributions, fitted with two components. The multiple exponential components of the outward- and inward-facing states likely represent a collection of conformations. For example, it has been shown that the HP2 gate must swing away from the transport domain for substrate (un)loading ${ }^{15,51}$. Thus, it can be hypothesized that the outward- and inward-facing states encompass conformational heterogeneity of HP2 and that the longer dwell components comprise such open or otherwise restructured gate states. Thus, unlike the outward-facing state, which has a $\sim 2 \mathrm{~s}$ dwell-time component in transport conditions, the intermediate state is always comparatively short-lived in both apo and transport conditions. The intermediate state was, however, on average, significantly longer-lived (297 ms vs $175 \mathrm{~ms}$ ), and was captured about two times more often $(548 / 2589=21 \%$ 
a

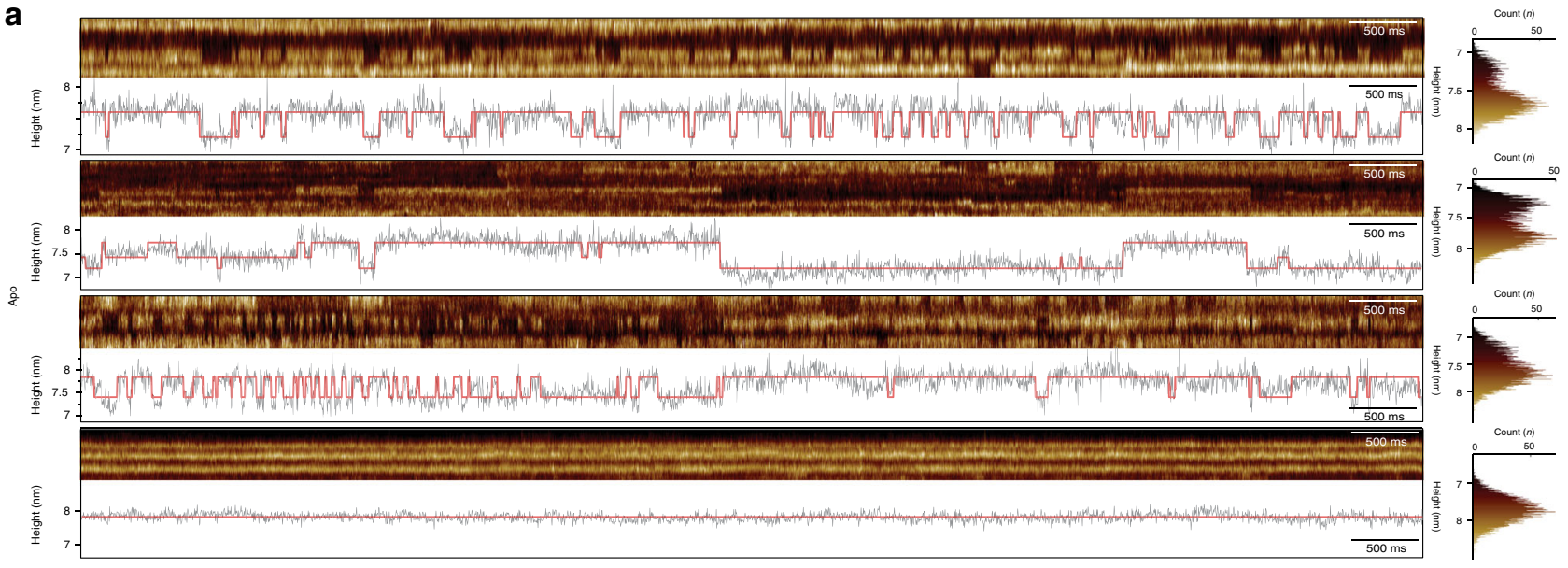

b
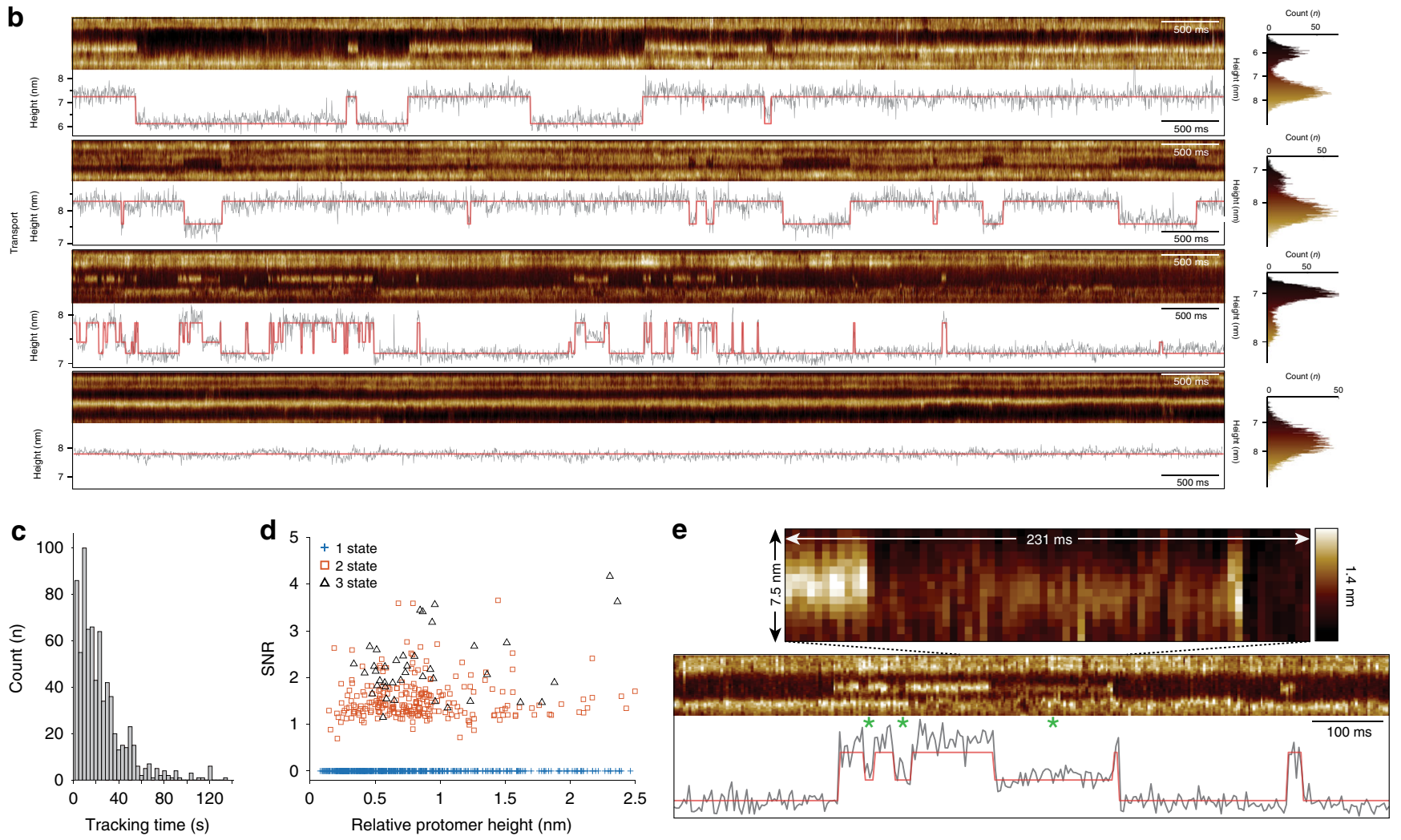

Fig. 3 Elevator dynamics of single unlabeled GIt $\mathbf{t}_{\mathbf{P h}}$ transporter domains. HS-AFM-LS raw data kymographs and height/time traces recorded in apo $\mathbf{a}$ and transport $\mathbf{b}$ conditions. All kymographs were recorded at $3.3 \mathrm{~ms}$ line acquisition speed. The height/time traces (gray) report the movements of the protomer in the center of the kymograph and are overlaid by the state-transition trace (red). The last kymograph and height/time trace in each condition shows traces of inactive protomers (see Table S2). Right: height distributions of the protomers under investigation. c Length distribution of GltPh transport domain tracking in HS-AFM-LS experiments $(n=762)$. d Signal-to-noise ratio (SNR) plotted against the protomer relative height over membrane in the outward-facing state for all analyzed 762 individual protomers. Traces assigned with 1, 2, and 3 amplitude states are represented by blue crosses, red squares, and black triangles, respectively. e A representative raw data kymograph and height/time trace (gray) overlaid by the idealized state-transition trace (red) of a transport domain visiting three states. Asterisks indicate intermediate state intervals. Top: pixel-by-pixel display of $7.5 \mathrm{~nm}$ lateral dimension and $231 \mathrm{~ms}$ raw data of the center (dashed lines) of the 3-state kymograph shown below, allowing the viewer to observe the SNR and pixel-statistics of HSAFM-LS. The full false color scale of the pixels shown is $1.4 \mathrm{~nm}$.

vs $838 / 7912=11 \%$ ) in the presence, compared to the absence of the substrates. Functionally, an intermediate state must be visited in each TM passage ${ }^{51}$. It is, however, not necessary that the transport domains stall in any intermediate position with a measurable dwell-time. Thus, the comparative shortness and rareness of the observed intermediate states in apo conditions suggest that most of the time, the transport domain undergoes rapid diffusive motions across the membrane. Longer dwell-times and increased observation frequency under transport conditions indicate that the loaded domain has a higher propensity to get stuck during the "elevator" movement; potentially in agreement with a recent cryo-EM study where an intermediate-outward state 
a

a Inward-facing state
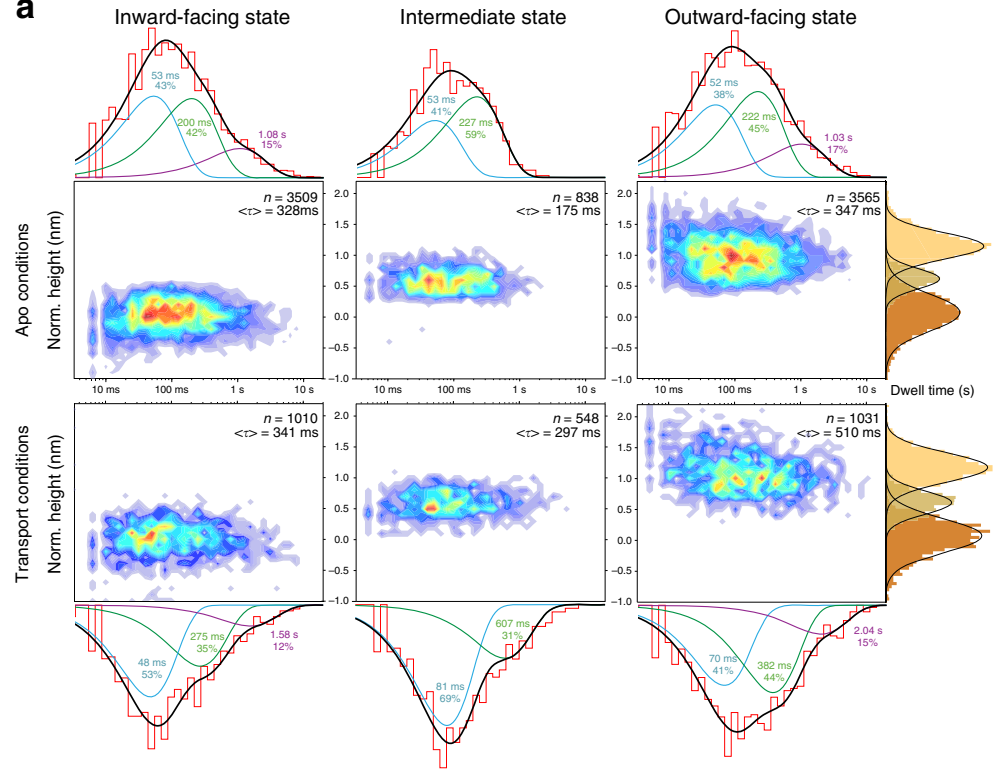

b
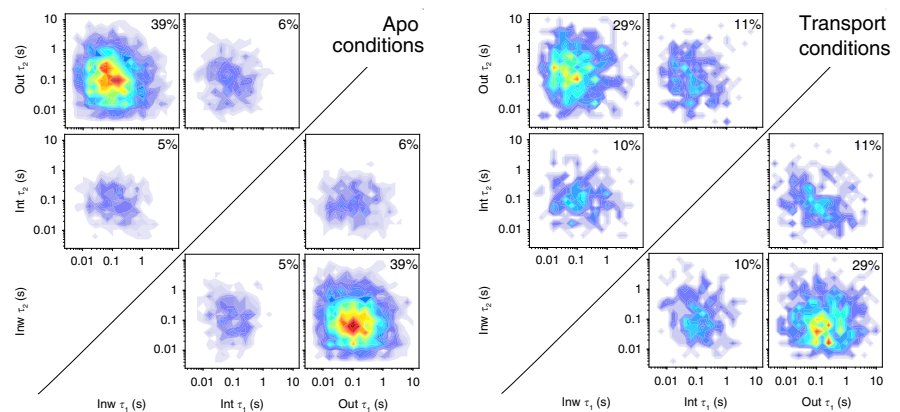

C

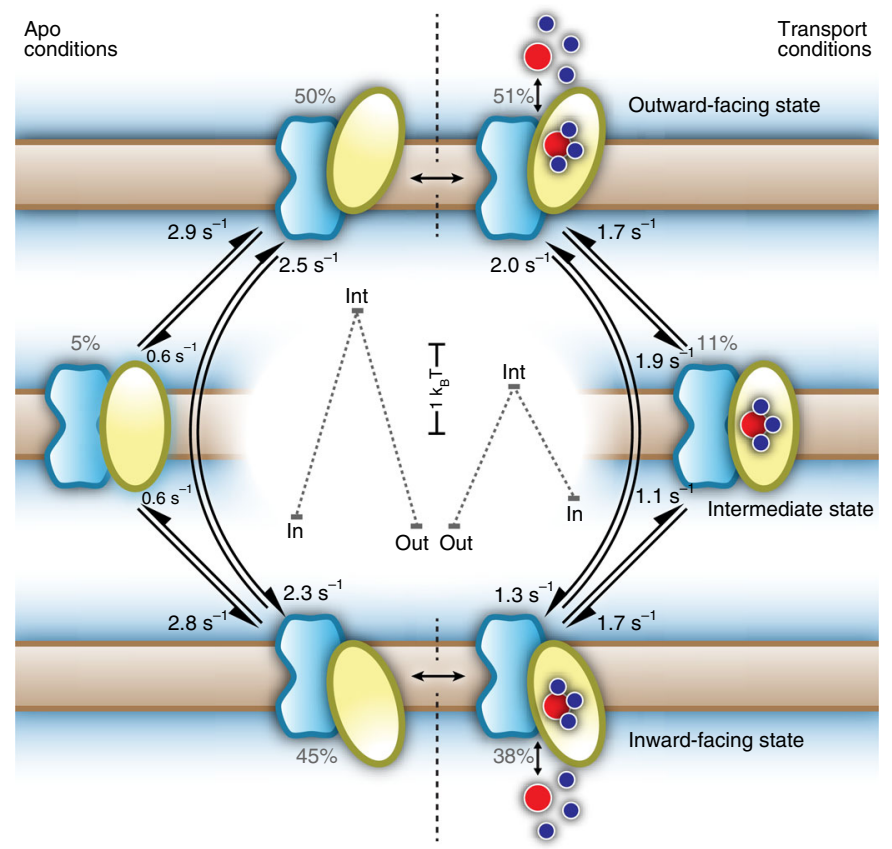

was found in presence of transport substrates ${ }^{23}$. Longer-lived intermediate states might comprise states where the transport domain laterally dissociates from the trimerization domain ${ }^{52}$. Overall, dynamic protomers showed TM cycling activity with average time constants of $1.4 \mathrm{~s}^{-1}$ in apo and $1.05 \mathrm{~s}^{-1}$ in transport conditions.
HS-AFM-LS height/time traces allow us to follow the sequence of movements of single protomers for extended periods and provides detailed information on the transport domain state occupancy (Table S2) and pathway probabilities (Table S3). In both the absence and presence of substrates, the transport domains most frequently ventured directly between the outward- 
Fig. 4 Dynamics of the $\mathbf{~ G l}_{\mathbf{P h}}$ transport cycle. a Density maps of dwell-times vs height of transport domains in the absence and presence of substrates, i.e. apo (top) and transport (bottom) conditions: inward- (left), intermediate (middle), and outward- (right) facing state dwell-times. All density maps and histograms (above and below the density maps) were logarithmically binned. The dwell-time histograms were fitted (black) with multiple exponential components (blue, green, and purple) and the corresponding exponential decay values and occurrence percentages are indicated. The transporter domain height distributions (far right) are well fitted by Gaussians. The total number of transitions $(n)$ and the average dwell-times $(\langle\tau\rangle)$ are indicated in the tope right of each panel. b State-transition tiles in apo (left) and transport (right) conditions, where the initial state and the final state are plotted on the $x$ - and $y$ axis, respectively. Inside the tiles: density maps of the initial- vs the final- dwell-time of each transition. c Schematic representation of the Glt $t_{\text {Ph }}$ transport cycle. HS-AFM-LS characterizes the membrane transitions in either apo- or transport conditions in independent experiments (separated by the black dashed line). Only a single trimerization domain (blue) and transport domain (yellow) are shown. Arrows indicate transport domain pathways. The numbers next to each arrow indicate the weighted rate constants (see "Methods") from one state to another. The percentages next to each state indicate the mean occupancy (see "Methods") of each state, from which the energy landscapes (central inset) are calculated (note 1: the energy barriers between states are not determined; note 2: the energy landscape only describes the dynamic protomers). Asp (red dot) and $\mathrm{Na}^{+}$(blue dots) associate and dissociate to/from the transport domain on either side of the membrane.

and inward-facing states, $78 \%$ and $58 \%$, respectively (Fig. $4 \mathrm{~b}$ ). The increased spatio-temporal resolution of HS-AFM-LS revealed furthermore that $22 \%$ and $42 \%$ of the protomer excursions went to or came from the intermediate state in the absence and presence of substrates, respectively.

The conditional distributions of the consecutive dwell-times of the initial and the final states can be plotted within each state transition tile (Fig. 4b). In apo conditions, these plots show no correlation between consecutive dwell-times. The dwell-time distributions are almost symmetric between tiles of reversedorder transitions, such as from the outward- to the inward- and from the inward- to the outward-facing states, proving that the HS-AFM imaging does not bias the transitions. Also, the outward-to-inward and inward-to-outward tiles appear almost symmetric intrinsically, because, in apo condition, the dwell-time distributions of the outward- and inward-facing states are very similar. In contrast, in transport conditions, the increased outward-facing dwell-times result in asymmetric distributions within the consecutive dwell-time tiles (Fig. 4b, right).

The extended height/time traces allow tracking protomers over several transition steps, providing further insights into the intermediate state kinetics. Specifically, we can distinguish between the situations when the intermediate state is visited measurably during the successful transitions between the outwardand inward-facing states and when it is visited during the failed attempts followed by a return to the starting state (Table S3). This analysis shows that mean dwell-times are pathway-specific. This property is particularly pronounced in transport conditions, but is also detected in apo conditions. We found that for the failed attempts, i.e., out $\rightarrow$ int $\rightarrow$ out or in $\rightarrow$ int $\rightarrow$ in, the initial mean state dwell-time was about two times longer than when the protomers crossed the membrane, i.e., out $\rightarrow$ int $\rightarrow$ in or in $\rightarrow$ int $\rightarrow$ out. Thermodynamically, the reverse movements of the failed excursions are expected. However, failed excursions (out $\rightarrow$ int $\rightarrow$ out or in $\rightarrow$ int $\rightarrow$ in) occur about two times more often than complete transitions (out $\rightarrow$ int $\rightarrow$ in or in $\rightarrow$ int $\rightarrow$ out). Moreover, the dwell-times of the starting states are longer for the failed compared to complete transitions (Table S3). These observations indicate a structural coupling of the outward- and inward-facing states with their neighboring intermediate state (Table S3, graphical summary).

The establishment of a detailed kinetic model comprising all kinetic sub-states (Fig. 4a) and the pathway-specific state dwelltimes and pathway probabilities (Table S3) is beyond the scope of this work, and would need many more hours of recording (we currently recorded 100 and 153 min of protomer kymographs in apo and transport conditions, respectively; Table S2). Instead, we can draw a simplified kinetic transport model with weighted rate constants (see "Methods") that recapitulates that the transitions between the outward- and inward-facing states can occur by multiple pathways (Fig. 4c). The corresponding energy landscapes highlight that the probability to transition through the intermediate states is higher in transport- compared to apo conditions (Fig. 4c, inset). The energy landscape further illustrates that the outward-facing state is favored in both apo and transport conditions, in agreement with our earlier HS-AFM imaging analysis. In addition, in control experiments with TBOA or saturating $\mathrm{Na}^{+}$, protomers were found stalled in the outwardfacing state (Fig. S6). This is in contrast to a recent cryo-EM study of $\mathrm{Glt}_{\mathrm{Tk}}{ }^{23}$, where in $\mathrm{Na}^{+}$-only conditions two of three protomers stalled in the inward-facing state. This discrepancy could be explained by either differences between these very similar proteins or differences related to the experimental environment. It is, however, notable that in the same study in transport conditions or in presence of TBOA, preference for the outwardfacing state has been found ${ }^{23}$, similar to our findings here. This kinetic model and energy landscape are, however, representing dynamic molecules only. A large fraction of molecules is quiescent in the outward-facing state during our experimental time windows, thus if those molecules would be included, the outward-facing state would be a far lower energy state in the cycle.

\section{Discussion}

While ion channel research uses single-molecule electrophysiology to characterize channel kinetics with single-molecule resolution, there is a paucity of similar approaches in transporter research ${ }^{53}$. smFRET $^{29}$ and HS-AFM imaging ${ }^{33}$ have provided solutions, however with much reduced bandwidth. Here, we introduce HS-AFM-LS ${ }^{37}$ for the investigation of singletransporter dynamics with temporal resolution comparable to single-channel recordings. This development allowed us here to detect the rapid translocation kinetics of the dynamic $\mathrm{Glt}_{\mathrm{Ph}}$ domains and revealed an intermediate state in the transport cycle. It will also be key for the study of mammalian EAATs that are significantly faster than $\mathrm{Glt}_{\mathrm{Ph}}{ }^{54}$. Based on our HS-AFM-LS data on membrane-embedded $\mathrm{Glt}_{\mathrm{Ph}}$, we extract kinetics which show that each state of the cycle englobes a family of structural substates which show distinct mean dwell-times. Perhaps most remarkably, we show that there are multiple transition pathways between the outward- to the inward-facing states, as computationally proposed ${ }^{52,55}$; establishing a detailed kinetic model comprising multiple pathways and intermediates is a future challenge. Readily, we see that in some of these pathways, an intermediate state is populated for over a hundred milliseconds, while in others, the translocation occurs without delays.

\section{Methods}

Protein purification. Glt $t_{\mathrm{Ph}}$ was expressed and purified as previously described ${ }^{12,27}$. Briefly, the protein was expressed in Escherichia coli DH10b strain with a 
C-terminal thrombin cleavage site and 8-His-tag. The isolated crude membranes were solubilized in a buffer containing $20 \mathrm{mM}$ Hepes, pH7.4, $200 \mathrm{mM} \mathrm{NaCl}$, $0.1 \mathrm{mM}$ L-aspartate supplemented with $40 \mathrm{mM}$ n-dodecyl $\beta$-D-maltopyranoside (DDM) for $2 \mathrm{~h}$ at $4{ }^{\circ} \mathrm{C}$. Solubilized transporters were then applied to immobilized metal affinity resin in the presence of $1 \mathrm{mM}$ DDM. The resin was washed in the same buffer supplemented with $40 \mathrm{mM}$ imidazole, subsequently $\mathrm{Glt}_{\mathrm{Ph}}$ was eluted in the presence of $250 \mathrm{mM}$ imidazole. The 8-His-tag was removed through thrombin digestion overnight, and the protein was further purified by size-exclusion chromatography in a buffer containing $10 \mathrm{mM}$ Hepes, pH7.4, $100 \mathrm{mM} \mathrm{NaCl}, 0.1 \mathrm{mM} \mathrm{L}$ aspartate, $0.4 \mathrm{mM}$ DDM. The protein was concentrated to $\sim 5 \mathrm{mg} / \mathrm{ml}$, flash frozen, and stored at $-80^{\circ} \mathrm{C}$. Protein concentration was determined by absorbance at $280 \mathrm{~nm}$ using an extinction co-efficient of $57,400 \mathrm{M}^{-1} \mathrm{~cm}^{-1}$ (per monomer).

Protein reconstitution. Purified Glt $t_{\mathrm{Ph}}$ was diluted to a concentration of $1 \mathrm{mg} / \mathrm{ml}$ in a buffer containing $10 \mathrm{mM}$ Tris, pH7.4, $100 \mathrm{mM} \mathrm{NaCl}, 10 \mathrm{mM} \mathrm{MgCl}_{2}$ supplemented with $0.05 \%$ DDM, and aliquoted into $50-\mu \mathrm{l}$ samples for reconstitution at various lipid-to-protein ratios (LPRs). Lipids, a 1,2-dioleoyl- sn-glycero-3phosphocholine (DOPC)/1,2-dioleoyl-sn-glycero- 3-phosphoethanolamine (DOPE)/1,2-dioleoyl-sn-glycero-3-phospho-L-serine (DOPS) 8:1:1 lipid mixture (all lipids from Avanti Polar Lipids), were pre-solubilized in 2\% DDM and added at LPRs between 0.5 and 1.0 (w:w). After $1 \mathrm{~h}$ of equilibration, $\sim 5 \mathrm{mg}$ of wet BioBeads (BioRad) were added to each reconstitution trial for detergent removal. After overnight incubation, BioBeads were removed from the sample and the reconstitutions checked by negative-stain electron microscopy for the presence of wellcontrasted (protein-packed) vesicles of size sufficiently large $(>100 \mathrm{~nm})$ for HSAFM analysis.

Sample preparation. A 2- $\mu$ d drop of the $\mathrm{Glt}_{\mathrm{Ph}}$-reconstituted vesicles was deposited on a $1.5 \mathrm{~mm}$ diameter freshly cleaved mica surface, which was glued with epoxy to a quartz sample stage. After $30 \mathrm{~min}$ incubation in a humid chamber, the sample was gently rinsed with imaging buffer and mounted in the HS-AFM fluid cell $^{33,56,57}$.

High-speed atomic force microscopy (HS-AFM). All images in this study were taken using a HS-AFM (SS-NEX, RIBM, Tsukuba, Japan) ${ }^{36}$ operated in amplitude modulation mode (with typical free and setpoint amplitudes, $A_{\text {free }}=1.0 \mathrm{~nm}$ and $A_{\text {set }}=0.9 \mathrm{~nm}$, respectively ${ }^{58,59}$ using optimized scan and feedback parameters. Ultrashort $(8 \mu \mathrm{m})$ cantilevers (USC-F1.2-k0.15, NanoWorld, Neuchatel, Switzerland) with nominal spring constant of $0.15 \mathrm{~N} / \mathrm{m}$, and resonance frequency of $\sim 650$ $\mathrm{kHz}$ and quality factor of $\sim 1.5$ in buffer, were used. In the presented experiments, four different buffer conditions were used. No substrate (apo) conditions: $20 \mathrm{mM}$ Tris-HCl, pH7.5, $150 \mathrm{mM} \mathrm{KCl}$; transport conditions ( $\mathrm{Na}^{+}$and Asp): $20 \mathrm{mM}$ Tris$\mathrm{HCl}, \mathrm{pH} 7.5,150 \mathrm{mM} \mathrm{NaCl}, 1 \mu \mathrm{M}$ Asp; saturating $\mathrm{Na}^{+}$conditions: $20 \mathrm{mM}$ Tris$\mathrm{HCl}, \mathrm{pH} 7.5,1 \mathrm{M} \mathrm{NaCl}$; and inhibitory conditions (blocker): $20 \mathrm{mM}$ Tris- $\mathrm{HCl}$, $\mathrm{pH} 7.5,150 \mathrm{mM} \mathrm{NaCl}, 1 \mathrm{mM}$ DL-TBOA. We investigated $\mathrm{Glt}_{\mathrm{Ph}}$ from two protein purifications from which we made $\sim 5$ reconstitutions, at $\sim 5$ LPRs (LPRs between 0.5 and $1.0(w: w))$ each

High-speed atomic force microscopy line scanning (HS-AFM-LS). To improve the temporal resolution for the characterization of transporter motions, the spatial dimensionality of data acquisition was reduced ${ }^{60}$. Disabling the slow-scan axis $(y$ direction), the temporal resolution is improved by a factor corresponding to the number of scan lines acquired in the image right before slow-scan axis disabling, typically $\sim 300$ times, e.g., from $1 \mathrm{~s}$ temporal resolution in HS-AFM movies to 3.3 $\mathrm{ms}$ line acquisition speed, i.e. $6.6 \mathrm{~ms}$ temporal resolution, in HS-AFM-LS kymographs. Importantly, alternating between HS-AFM image and HS-AFM-LS kymograph acquisition does not change the physics of the data acquisition, in terms of tip oscillation, applied forces, actual tip velocity with respect to the sample, and feedback operation. The noise in HS-AFM-LS traces acquired on pure lipid bilayers of the same composition as the protein is reconstituted in the subAngstrom range ${ }^{60}$ (Fig. S8). If anything, HS-AFM-LS operates slightly better than HS-AFM imaging, because imprecisions from scanning the slow-scan axis are eliminated.

Data analysis. HS-AFM-LS kymographs were contrast adjusted, drift corrected, and assembled by routines and home-written analysis software in Igor Pro Software (WaveMetrics, Lake Oswego, OR, USA) and ImageJ (Fig. S3). To analyze HSAFM-LS kymographs, (1) each protomer was tracked, (2) its signal transferred into a height/time trace, and (3) analyzed by a state detection algorithm using MATLAB (Matlab, Mathworks, Natick, USA). In brief, a 1D tracking algorithm was applied to the kymographs by low-pass filtering the time axis allowing the automatic detection of each protomer peak position over time. To allow tracking of protomers over extended inward-facing periods a track-linking algorithm is used that "looks forward in time" for the protomer to reappear at its position. The tracking information is then used to read the accurate height/time traces of each protomer from the unfiltered kymograph. It is possible that a protomer is lost through drift and later tracked again-such traces would be counted as two individual protomers. To determine the number of states and state transitions, we adapted the Step Transition and State Identification (STaSI) ${ }^{61}$ algorithm developed for discrete single-molecule data analysis for our HS-AFM-LS data ${ }^{60}$. The STaSI algorithm detects step transitions using Student's $t$ test and then groups segments by hierarchical clustering. The optimum number of states is suggested by weighing between the complexity of the model and the goodness of the fit (minimum description length) to find the simplest model with the least fitting error. We extensively tested the STaSI algorithm by generating traces with two or three states and overlaid them with large amounts of noise (Fig. S4). This provided us with an objective assessment of the state-transition detection and state-number assignment accuracy of the algorithm, because we knew precisely the signal of the simulated trace without noise addition. In addition, the results were compared against another leading single-molecule state detection algorithm (vbFRET). The data showed that the STaSI algorithm outperformed vbFRET for the 3-state assignment particularly in the range of SNR we are dealing with. Both algorithms performed similarly well for the 2-state assignment.

The signal-to-noise ratio (SNR) of height/time traces. The SNR of each height/ time trace is calculated by dividing the height difference between the fitted values of the outward- and inward-facing states $\Delta h$ (out-in) with the standard deviation of the noise (on each state) in the trace $\operatorname{std}_{(\text {state) }}$ :

$$
\mathrm{SNR}=\Delta h_{(\text {out-in) }} / \operatorname{std}_{(\text {state })} \text {. }
$$

Dwell-time representation and fitting. For clarity of illustration (Fig. 4a), the dwell-times plots were displayed following the Sigworth method ${ }^{50}$, a standard to display dwell-time distributions of single-channel recordings. Dwell-time distributions were fitted with multi-exponential fits and reduced $\chi^{2}$ tests were used to avoid overfitting of the dwell-time distributions.

Kinetic scheme representation. For illustration of a simplified kinetic scheme (Fig. 4c), weighted rate constants $k^{\prime}$ for each transition were calculated from average dwell-times of each state $\left\langle\tau_{(\text {state }-n)}\right\rangle$ weighted by the relative occurrence $n_{\text {(state }-n \rightarrow \text { state }-m)}$ of the transition:

$k_{(\text {state-1 } \rightarrow \text { state-2) }}^{\prime}=1 /\left\langle\tau_{(\text {state-1) }}\right\rangle \times n_{(\text {state-1 } \rightarrow \text { state-2) }} / n_{(\text {state-1 } \rightarrow \text { state-2) }}+n_{(\text {state-1 } \rightarrow \text { state-3) }}$.

To derive the state distribution probabilities (Table S2 and Fig. 4c), the average probabilities $\rho_{(\text {state }-n)}$ of finding an active protomer in a given state was calculated from average dwell-times of each state $\left\langle\tau_{(\text {state }-n)}\right\rangle$ and the relative occurrence $n_{(\text {state }-n)}$ of the state:

$$
\begin{aligned}
\rho_{\text {(state-1) }}=n_{(\text {state-1) }}\left\langle\tau_{\text {(state-1) }}\right\rangle / n_{(\text {state-1) }} \times\left\langle\tau_{\text {(state-1) }}\right\rangle & +n_{\text {(state-2) }} \times\left\langle\tau_{\text {(state-2) }}\right\rangle \\
+ & +n_{\text {(state- } 3)} \times\left\langle\tau_{\text {(state-3) }}\right\rangle .
\end{aligned}
$$

From the state distribution probabilities (Table S2 and Fig. $4 \mathrm{c}$ ), the energy differences between states $\Delta G_{(\text {state }-n \hookleftarrow \text { state }-m)}$ was calculated:

$$
\Delta G_{(\text {state }-n \hookleftarrow \text { state }-m)}=k_{B} T \times \ln \left(\rho_{(\text {state }-n)} / \rho_{(\text {state }-m)}\right) .
$$

Reporting summary. Further information on research design is available in the Nature Research Reporting Summary linked to this article.

\section{Data availability}

Data supporting the findings of this manuscript are available from the corresponding author upon reasonable request. Source data are provided with this paper.

\section{Code availability}

MATLAB codes used for data analysis are provided in Supplementary Information file Supplementary MATLAB Codes.

Received: 6 April 2020; Accepted: 16 September 2020; Published online: 06 October 2020

\section{References}

1. Beart, P. \& O'shea, R. Transporters for L-glutamate: an update on their molecular pharmacology and pathological involvement. Br. J. Pharmacol. 150, 5-17 (2007)

2. Danbolt, N. C. Glutamate uptake. Prog. Neurobiol. 65, 1-105 (2001).

3. Coincon, $\mathrm{M}$. et al. Crystal structures reveal the molecular basis of ion translocation in sodium/proton antiporters. Nat. Struct. Mol. Biol. 23, 248-255 (2016)

4. Landreh, M. et al. Integrating mass spectrometry with MD simulations reveals the role of lipids in $\mathrm{Na}(+) / \mathrm{H}(+)$ antiporters. Nat. Commun. 8, 13993 (2017). 
5. Fairman, W., Vandenberg, R., Arriza, J., Kavanaught, M. \& Amara, S. An excitatory amino-acid transporter with properties of a ligand-gated chloride channel. Nature 375, 599-603 (1995).

6. Fuchs, B. C. \& Bode, B. P. Amino acid transporters ASCT2 and LAT1 in cancer: Partners in crime? Semin. Cancer Biol. 15 254-266 (2005).

7. Veruki, M. L., Mørkve, S. H. \& Hartveit, E. Activation of a presynaptic glutamate transporter regulates synaptic transmission through electrical signaling. Nat. Neurosci. 9, 1388-1396 (2006).

8. Levy, O., De la Vieja, A. \& Carrasco, N. The Na+/I- symporter (NIS): recent advances. J. Bioenerg. Biomembranes 30, 195-206 (1998).

9. Zerangue, N. \& Kavanaugh, M. P. Flux coupling in a neuronal glutamate transporter. Nature 383, 634-637 (1996).

10. Canul-Tec, J. C. et al. Structure and allosteric inhibition of excitatory amino acid transporter 1. Nature 544, 446-451 (2017).

11. Garaeva, A. A. et al. Cryo-EM structure of the human neutral amino acid transporter ASCT2. Nat. Struct. Mol. Biol. 25, 515-521 (2018).

12. Yernool, D., Boudker, O., Jin, Y. \& Gouaux, E. Structure of a glutamate transporter homologue from Pyrococcus horikoshii. Nature 431, 811-818 (2004).

13. Boudker, O. \& Verdon, G. Structural perspectives on secondary active transporters. Trends Pharmacol. Sci. 31, 418-426 (2010).

14. Boudker, O., Ryan, R. M., Yernool, D., Shimamoto, K. \& Gouaux, E. Coupling substrate and ion binding to extracellular gate of a sodium-dependent aspartate transporter. Nature 445, 387-393 (2007).

15. Huang, Z. J. \& Tajkhorshid, E. Dynamics of the extracellular gate and ionsubstrate coupling in the glutamate transporter. Biophysical J. 95, 2292-2300 (2008).

16. Shrivastava, I. H., Jiang, J., Amara, S. G. \& Bahar, I. Time-resolved mechanism of extracellular gate opening and substrate binding in a glutamate transporter. J. Biol. Chem. 283, 28680-28690 (2008).

17. Grazioso, G. et al. Investigating the mechanism of substrate uptake and release in the glutamate transporter homologue Glt $_{\mathrm{Ph}}$ through metadynamics simulations. J. Am. Chem. Soc. 134, 453-463 (2012).

18. Heinzelmann, G., Bastug, T. \& Kuyucak, S. Mechanism and energetics of ligand release in the aspartate transporter $\mathrm{Glt}_{\mathrm{Ph}}$. J. Phys. Chem. B 117, 5486-5496 (2013).

19. Verdon, G. \& Boudker, O. Crystal structure of an asymmetric trimer of a bacterial glutamate transporter homolog. Nat. Struct. Amp; Mol. Biol. 19, 355-357 (2012).

20. Zomot, E. \& Bahar, I. Intracellular gating in an inward-facing state of aspartate transporter $\mathrm{Glt}_{\mathrm{Ph}}$ is regulated by the movements of the helical hairpin HP2. J. Biol. Chem. 288, 8231-8237 (2013).

21. Garaeva, A. A., Guskov, A., Slotboom, D. J. \& Paulino, C. A one-gate elevator mechanism for the human neutral amino acid transporter ASCT2. Nat. Commun. 10, 3427 (2019)

22. Groeneveld, M. \& Slotboom, D.-J. Na+: aspartate coupling stoichiometry in the glutamate transporter homologue Glt $\mathrm{Ph}_{\mathrm{Ph}}$. Biochemistry 49, 3511-3513 (2010).

23. Arkhipova, V., Guskov, A. \& Slotboom, D. J. Structural ensemble of a glutamate transporter homologue in lipid nanodisc environment. Nat. Commun. 11, 998 (2020)

24. Ryan, R. M., Compton, E. L. R. \& Mindell, J. A. Functional characterization of a $\mathrm{Na}+$-dependent aspartate transporter from Pyrococcus horikoshii. J. Biol. Chem. 284, 17540-17548 (2009).

25. Abramson, J. et al. Structure and mechanism of the lactose permease of Escherichia coli. Science 301, 610-615 (2003).

26. Krishnamurthy, H. \& Gouaux, E. X-ray structures of LeuT in substrate-free outward-open and apo inward-open states. Nature 481, 469-474 (2012).

27. Reyes, N., Ginter, C. \& Boudker, O. Transport mechanism of a bacterial homologue of glutamate transporters. Nature 462, 880-885 (2009).

28. Akyuz, N., Altman, R. B., Blanchard, S. C. \& Boudker, O. Transport dynamics in a glutamate transporter homologue. Nature 502, 114-118 (2013).

29. Akyuz, N. et al. Transport domain unlocking sets the uptake rate of an aspartate transporter. Nature 518, 68-73 (2015).

30. Erkens, G. B., Hänelt, I., Goudsmits, J. M., Slotboom, D. J. \& van Oijen, A. M. Unsynchronised subunit motion in single trimeric sodium-coupled aspartate transporters. Nature 502, 119-123 (2013).

31. Hänelt, I., Wunnicke, D., Bordignon, E., Steinhoff, H.-J. \& Slotboom, D. J. Conformational heterogeneity of the aspartate transporter Glt $\mathrm{ph}_{\mathrm{p}}$. Nat. Struct. Mol. Biol. 20, 210-214 (2013).

32. Georgieva, E. R., Borbat, P. P., Ginter, C., Freed, J. H. \& Boudker, O. Conformational ensemble of the sodium-coupled aspartate transporter. Nat. Struct. Mol. Biol. 20, 215-221 (2013).

33. Ruan, Y. et al. Direct visualization of glutamate transporter elevator mechanism by high-speed AFM. Proc. Natl Acad. Sci. USA 114, 1584-1588 (2017).

34. Mulligan, C. et al. The bacterial dicarboxylate transporter VcINDY uses a twodomain elevator-type mechanism. Nat. Struct. Mol. Biol. 23, 256-263 (2016).
35. Garaeva, A. A. \& Slotboom, D. J. Elevator-type mechanisms of membrane transport. Biochem. Soc. Trans. https://doi.org/10.1042/BST20200290 (2020).

36. Ando, T. et al. A high-speed atomic force microscope for studying biological macromolecules. Proc. Natl Acad. Sci. 98, 12468-12472 (2001).

37. Heath, G. R. \& Scheuring, S. High-speed AFM height spectroscopy reveals $\mu$ sdynamics of unlabeled biomolecules. Nat. Commun. 9, 4983 (2018).

38. McIlwain, B. C., Vandenberg, R. J. \& Ryan, R. M. Transport rates of a glutamate transporter homologue are influenced by the lipid bilayer. J. Biol. Chem. 290, 9780-9788 (2015)

39. Benediktsson Adrienne, $M$. et al. Neuronal activity regulates glutamate transporter dynamics in developing astrocytes. Glia 60, 175-188 (2011).

40. Otis, T. S. \& Dodson, P. D. Transporter Proteins in Neurons and Glia. 11591166 https://doi.org/10.1016/B978-008045046-9.01211-0 (Encyclopedia of Neuroscience, 2010).

41. Kodera, N., Yamamoto, D., Ishikawa, R. \& Ando, T. Video imaging of walking myosin V by high-speed atomic force microscopy. Nature 468, 72-76 (2010).

42. Viani, M. B. et al. Probing protein-protein interactions in real time. Nat. Struct. Biol. 7, 644-647 (2000).

43. Zhou, W. et al. Large-scale state-dependent membrane remodeling by a transporter protein. eLife 8, e50576 (2019)

44. Huang, Y. et al. Use of paramagnetic 19F NMR to monitor domain movement in a glutamate transporter homolog. Nat. Chem. Biol. https://doi.org/10.1038/ s41589-020-0561-6 (2020).

45. Stolzenberg, S., Khelashvili, G. \& Weinstein, H. Structural intermediates in a model of the substrate translocation path of the bacterial glutamate transporter homologue $\mathrm{Glt}_{\mathrm{ph}}$. J. Phys. Chem. B 116, 5372-5383 (2012),

46. Chen, I. et al. Glutamate transporters contain a conserved chloride channel with two hydrophobic gates. bioRxiv. https://doi.org/10.1101/ 2020.05.25.115360 (2020)

47. Wang, J., Albers, T. \& Grewer, C. Energy landscape of the substrate translocation equilibrium of plasma-membrane glutamate transporters. $J$ Phys. Chem. B 122, 28-39 (2018).

48. Wang, J. et al. Crystal structure of a bacterial homologue of SWEET transporters. Cell Res. 24, 1486-1489 (2014).

49. Perez, C., Koshy, C., Yildiz, Ö. \& Ziegler, C. Alternating-access mechanism in conformationally asymmetric trimers of the betaine transporter BetP. Nature 490, 126-130 (2012).

50. Sigworth, F. J. \& Sine, S. M. Data transformations for improved display and fitting of single-channel dwell time histograms. Biophys. J. 52, 1047-1054 (1987).

51. Drew, D. \& Boudker, O. Shared molecular mechanisms of membrane transporters. Annu. Rev. Biochem. 85, 543-572 (2016).

52. Machtens, J.-P. et al. Mechanisms of anion conduction by coupled glutamate transporters. Cell 160, 542-553 (2015)

53. McManus, O. B., Weiss, D. S., Spivak, C. E., Blatz, A. L. \& Magleby, K. L. Fractal models are inadequate for the kinetics of four different ion channels. Biophysical J. 54, 859-870 (1988).

54. Wadiche, J. I., Arriza, J. L., Amara, S. G. \& Kavanaugh, M. P. Kinetics of a human glutamate transporter. Neuron 14, 1019-1027 (1995).

55. Jiang, T. et al. Computational dissection of membrane transport at a microscopic level. Trends Biochemical Sci. 45, 202-216 (2020).

56. Ruan, Y. et al. Structural titration of receptor ion channel GLIC gating by HS AFM. Proc. Natl Acad. Sci. USA 115, 10333-10338 (2018).

57. Miyagi, A., Chipot, C., Rangl, M. \& Scheuring, S. High-speed atomic force microscopy shows that annexin $\mathrm{V}$ stabilizes membranes on the second timescale. Nat. Nanotechnol. 11, 783-790 (2016).

58. Miyagi, A. \& Scheuring, S. Automated force controller for amplitude modulation atomic force microscopy. Rev. Sci. Instrum. 87, 053705 (2016)

59. Lin, Y.-C. et al. Force-induced conformational changes in PIEZO1. Nature 573, 230-234 (2019).

60. Heath, G. R. \& Scheuring, S. High-speed AFM height spectroscopy reveals micros-dynamics of unlabeled biomolecules. Nat. Commun. 9, 4983 (2018).

61. Shuang, B. et al. Fast step transition and state identification (STaSI) for discrete single-molecule data analysis. J. Phys. Chem. Lett. 5, 3157-3161 (2014).

\section{Acknowledgements}

This research was supported by the grant NIH NINDS RO1NS110790 (to S.S.).

\section{Author contributions}

S.S. and O.B. designed the study. T.R.M. performed protein reconstitution, HS-AFM analysis, and data analysis. G.R.H. customized MATLAB algorithms and analyzed the data. S.S. supervised protein reconstitution, HS-AFM analysis, and data analysis. G.H.M.H. expressed and purified protein. O.B. supervised protein expression and purification. T.R.M. and S.S. wrote the manuscript with input from all authors. 


\section{Competing interests}

The authors declare no competing interests.

\section{Additional information}

Supplementary information is available for this paper at https://doi.org/10.1038/s41467020-18811-z.

Correspondence and requests for materials should be addressed to S.S.

Peer review information Nature Communications thanks the anonymous reviewers for their contributions to the peer review of this work.

Reprints and permission information is available at http://www.nature.com/reprints

Publisher's note Springer Nature remains neutral with regard to jurisdictional claims in published maps and institutional affiliations. (c) (i) Open Access This article is licensed under a Creative Commons Attribution 4.0 International License, which permits use, sharing, adaptation, distribution and reproduction in any medium or format, as long as you give appropriate credit to the original author(s) and the source, provide a link to the Creative Commons license, and indicate if changes were made. The images or other third party material in this article are included in the article's Creative Commons license, unless indicated otherwise in a credit line to the material. If material is not included in the article's Creative Commons license and your intended use is not permitted by statutory regulation or exceeds the permitted use, you will need to obtain permission directly from the copyright holder. To view a copy of this license, visit http://creativecommons.org/ licenses/by/4.0/.

(C) The Author(s) 2020 